\title{
Effect of Chemical Composition of Nutritive Medium and Explant Size Over Androgenetic Response in Microspore Culture of Brassica oleracea $\mathbf{L}$.
}

\author{
TINA OANA CRISTEA*, GABRIEL-ALIN IOSOB, CREOLA BREZEANU, \\ PETRE MARIAN BREZEANU \\ Vegetable Research and Development Station Bacau, 220 Calea Barladului, 600388, Bacau, Romania
}

\begin{abstract}
The dimension of the bud is a key factor for the orientation of microspore culture and the success of obtaining double haploid plants as it is a strong correlation between bud size and the developmental stages of microspores, and it is specific for each plant species and genotype. Our study was focused to determine the correlation between morphological characteristics, namely floral bud size and specific microspore developmental stages in order to determine the proper size, suitable for a successful protocol of obtaining double haploid plants in Brassica oleracea var. italica. Thus, we tested four bud sizes ranging from 2.0 to $4.0 \mathrm{~mm}$ measured from the base to the tip of the bud. After the statistical analysis of the results it can be emphasized that the best results were obtained in the case of using as a source of microspores the flower buds with the size between 3.1-3.5 mm. At this dimension, the share of microspores in the uninucleate stage, predominantly in the late uninucleate stage, is $90 \%$, thus ensuring a homogeneous population of microspores in the optimum stage of development. Their evolution is predominantly embryogenic, the percentage of microspores following the gametophytic pathway is reduced, by only $9.12 \%$.
\end{abstract}

Keywords: haploid, embryogenesis, floral, bud, vitro

\section{Introduction}

One of the main applications of plant biotechnology is in haploid plant breeding and this is due to the fact that is far more efficient than conventional plant breeding for the generation of diploid homozygous pure lines. These lines can be produced in a single generation by haploid in vitro culture, while several generations of inbreeding are required using traditional breeding. Usually for producing haploid plants anther culture was employed, but now-a-days a new method proved to be superior - the cultures of microspores rather than the whole anther [1-3].

Experimental haploidy in vitro, is a valuable tool that allows to accelerate breeding programs by increasing the so-called "gene pool"- the basis of genes available for breeding programs, as well as facilitating the selection of useful agronomic features [1]. Double haploid plants represent absolutely pure lines. Such a population phenotypically exhibits all the genetic variability of previous crosses. Thus, they represent a valuable breeding material for obtaining heterozygosity in all crop plants that reproduce sexually [2-5].

The production of haploid and doubled haploid plants using microspore culture provide the opportunity of obtaining homozygous lines within a shorter time period and with less labor and cost requirement as compared to the classic breeding techniques [3].

Most of the commercial crops of Brassica are F1 hybrids because they ensure a high degree of uniformity and productivity of the crops, as well as the protection of the copyright and the market of the private seed producing companies $[6,7]$.

The isolated microspore culture has the remarkable quality of allowing alternation of generations in the life cycle of angiosperms, is the switch between gametophytic and sporophytic haploid generations, ending with obtaining a sporophyte with a gamete number of chromosomes [8].

$\overline{\text { *email: tinaoana@yahoo.com }}$ 
The culture of microspores isolated on in vitro media has special implications in many fields: genetics and breeding, biochemical and physiological studies, induction and selection of mutants, genetic transformation, germplasm conservation etc. $[5,6]$. Completely homozygous plants from heterozygous parents can be obtained in this way in a single generation, compared to the classic way of improvement - by self-pollination, where at least 5-6 generations are needed [9].

Compared to the androgenesis generated via the culture of anthers, the method of obtaining dihaploid plants through microspore culture is evidenced by the possibility of obtaining a much larger number of such haploid embryos and neoplants [10].

The success of microspore cultures, as in the case of anthers, depends on a combination of intrinsic and extrinsic factors. In order to obtain a positive androgenetic response, it is necessary to follow some steps which mainly concern three stages: 1 . donor plant; 2 . - the period between the time of harvesting the flower buds and the microspore culture; 3. incubation period [9-11].

\section{Materials and methods}

\subsection{Plant growth conditions and biologic material}

The mother plants are grown in plastic pots $20 \mathrm{~cm}$ in diameter in greenhouses up to the stage of 10 leaves. Arrived at this stage, the plants are vernalized for 90 days under photoperiod conditions of $16 \mathrm{~h}$ with photosynthetic active radiation of about $60 \mu \mathrm{mol} \mathrm{m}^{-2} \mathrm{~s}^{-1}$, after vernalization they are passed under conditions of temperature of $15^{\circ} \mathrm{C}$ during the light period and $10^{\circ} \mathrm{C}$ during dark.

Plant fertilization is done weekly with liquid fertilizer (N: P: K - 20:10:20). Spraying with pesticides or fungicides is avoided because it affects the reactivity of the microspores, by taking preventive measures: decrease of humidity in the growth chamber, rapid removal of dead leaves etc.

The flower buds are excised with a portion of the stem, to avoid their oxidation and placed in Petri dishes on filter paper moistened in the refrigerator. Operations from bud harvesting to microspore isolation should be performed quickly to avoid degradation. In order to determine the optimum stage of harvesting of the flower buds, they were divided into 4 size classes, each of them constituting an experimental variant, realized in 3 repetitions (Table 1).

The excised buds were surface sterilized in $0.1 \%$ mercuric chloride (w/v) for $15 \mathrm{~min}$, followed by rinsing in sterile distilled water for 3 to 4 times.

The basal cultivation medium was $1 / 2 \mathrm{NLN}-13$ as decribed in literature), as it follows - the quantities

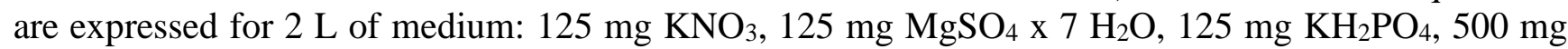
$\mathrm{Ca}\left(\mathrm{NO}_{3}\right)_{2} \times 4 \mathrm{H}_{2} \mathrm{O}, 40 \mathrm{mg}$ Fe EDTA, 800 L-Glutamine, $30 \mathrm{mg}$ Gluthatione, $100 \mathrm{mg}$ myo-inositol, 100 mg L-serine, For microelements NLN (1000x): $83 \mathrm{mg} \mathrm{Kl}, 1000 \mathrm{mg} \mathrm{H}_{3} \mathrm{BO}_{3}, 2500 \mathrm{mg} \mathrm{MnSO}_{4} \mathrm{x}_{4} \mathrm{H}_{2} \mathrm{O}$, $1000 \mathrm{mg} \mathrm{ZnSO}_{4} \times 7 \mathrm{H}_{2} \mathrm{O}, 25 \mathrm{mg} \mathrm{Na} 2 \mathrm{MoO}_{4} \times 2 \mathrm{H}_{2} \mathrm{O}, 2.5 \mathrm{mg} \mathrm{Cu}_{2} \mathrm{SO}_{4} \times 5 \mathrm{H}_{2} \mathrm{O}, 2.5 \mathrm{mg} \mathrm{CoCl} 2$ × $6 \mathrm{H}_{2} \mathrm{O}$ and for vitamines NLN (1000x) - $100 \mathrm{~mL}$ had the following composition: $50 \mathrm{mg}$ Thiamine $\mathrm{HCl}, 500 \mathrm{mg}$ Nicotinic acid, $50 \mathrm{mg}$ Pyridoxine $\mathrm{HCl}, 200 \mathrm{mg}$ Glycine, $5 \mathrm{mg}$ Biotin and $50 \mathrm{mg}$ Folic acid. To each medium $1 \mathrm{~mL}$ NLN vitamines (from $1000 \mathrm{x}$ stock solution), $1 \mathrm{~mL}$ NLN microsalts (from 1000x stock solution), and $260 \mathrm{~g}$ Sucrose (13\%) were added. The $p \mathrm{H}$ was adjusted to 5.8 and the sterilization of medium was made by filter sterilisation.

Table 1. Experimental variants tested

\begin{tabular}{|c|c|c|}
\hline Nr. crt. & Variant & Floral bud size \\
\hline 1. & V1 & $2.0-2.5 \mathrm{~mm}$ \\
\hline 2. & $\mathrm{~V} 2$ & $2.6-3.0 \mathrm{~mm}$ \\
\hline 3. & $\mathrm{~V} 3$ & $3.1-3.5 \mathrm{~mm}$ \\
\hline 4. & $\mathrm{~V} 4$ & $3.6-4 \mathrm{~mm}$ \\
\hline
\end{tabular}

\subsection{Microspore culture}

Sterile buds are transferred into $50 \mathrm{~mL}$ vials with $5 \mathrm{~mL}$ NLN medium [3] containing $13 \mathrm{~g}$ of sucrose. Microspores are released into the culture medium by lightly crushing the shoots with the plunger from a 20 or $50 \mathrm{~mL}$ syringe. The operation should be done carefully so as not to affect the microspores, which 
would result in a very small number of somatic embryos. The suspension is filtered through a sterile 40 $\mu \mathrm{m}$ nylon mesh and the filtrate was centrifuged $3 \mathrm{~min}$ at $200 \mathrm{~g}$. The supernatant is discarded and pellet is re-suspended in $10 \mathrm{~mL}$ medium and centrifuged again. The procedure is repeated three times. Finally, the microspores are suspended in 1-2 mL of NLN medium and plated in petri dishes with a density adjusted to $1 \times 10^{4}$ microspores $/ \mathrm{mL}$. The cultures are incubated in darkness at $33^{\circ} \mathrm{C}$ for three days and then each microspore suspension was centrifuged at $500 \mathrm{rpm}$ for $5 \mathrm{~min}$, and the microspores were washed with culture medium. The microspores are resuspended in 1-2 $\mathrm{mL}$ of medium and the culture plates are incubated to $25^{\circ} \mathrm{C}$ in dark for four weeks.

\subsection{Plant regeneration}

After 4 weeks of culture, each normal cotyledonary embryo (with well developed radicle, hypocotyl and two symmetrical cotyledons) was transferred to regeneration medium B5 (Gamborg 1968) supplemented with PGRs - BAP- $8.9 \mu \mathrm{M}$ and NAA $2.7 \mu \mathrm{M}, 2 \%$ sucrose and $0.08 \%$ agar-agar. The cultures were grown in a growth chamber at $25^{\circ} \mathrm{C}$ under a $16 / 8 \mathrm{~h}$ (day/night) photoperiod. Eight weeks after embryo transfer to the regeneration medium, plantlets with 2-5 leaves were transplanted to soil and the pots were covered with transparent vinyl for 2 weeks to allow acclimatization. The potted plantlets were grown in a growth room under the conditions described above for the flower-bud donor plants.

\subsection{Cytological studies}

Cytological studies we accomplished using the FDA (fluorescein diacetate) staining squash method for the screening of their viability during the early period after inoculation. 150-300 $\mu \mathrm{L}$ suspension with micropores is transferred in an Eppendorf tube. The volume is completed with culture medium until 1 $\mathrm{mL}$ and $1 \mathrm{~mL}$ stock solution of FDA medium is added. After $2 \mathrm{~min}$ the suspension is centrifugated and the pellet is removed. $10 \mu \mathrm{L}$ of suspension is used for squash sampling and observed under UV filter microscope HUND 600. The bright green cells are recorded and utilised for statistical analysis. The number of viable microspores in different stages was counted in randomly selected visual areas of the microscope in four replications per sample.

\subsection{Statistical analysis}

The experiments were accomplished in three replications, each one containing five plates per variant. The viability of microspores and the mean number of embryo per variant was recorded. The data were analyzed by ANOVA (analysis of variance). The means were compared using the Duncan multiple comparison test at $\mathrm{P}<0.05$.

\section{Results and discussions}

Determining the correlation between morphological characteristics, namely floral bud size and specific microspore developmental stages is a first step of the experiments, and along with improving the percentage of microspores with embryogenic capacity, supporting the development of embryogenic structures, and the formation of embryos are processes under the influence of numerous synergistic factors, their cumulative effect leading to increased efficiency of androgenesis microspores. The results are synthesized in Table 2 .

Table 2. The results obtained regarding the correlation of the size of the flower buds and the development stage of the microspores

\begin{tabular}{|c|c|c|c|c|c|}
\hline \multirow{2}{*}{ Variant } & \multicolumn{5}{|c|}{ Development stage } \\
\cline { 2 - 6 } & tetrade & Early uninucleated & $\begin{array}{c}\text { medium } \\
\text { muninucleated }\end{array}$ & late uninucleated & binucleated \\
\hline V1 & $51.2 \pm 2.9$ & $18.6 \pm 3.2$ & $11.3 \pm 4.6$ & $7.2 \pm 1.1$ & $0 \pm 0$ \\
\hline V2 & $27.8 \pm 3.1$ & $28.6 \pm 2.2$ & $15.3 \pm 1.1$ & $16.3 \pm 1.2$ & $1.9 \pm 0.2$ \\
\hline V3 & $1.1 \pm 0.3$ & $22.2 \pm 2.6$ & $32.3 \pm 1.4$ & $35.5 \pm 1.7$ & $2.8 \pm 0.1$ \\
\hline V4 & $0 \pm 0$ & $1.1 \pm 0.1$ & $19.20 \pm 2.4$ & $31.50 \pm 3.1$ & $39.30 \pm 3.4$ \\
\hline
\end{tabular}


Following the statistical processing of the data resulting from the determination of the stage of development of the microspores by the DAPI coloring technique and their correlation with the size of the flower buds, the following conclusions could be extracted:

- in the case of V1 variant - the size of the buds $2.0-2.5 \mathrm{~mm}, 51.2 \%$ of the microspores are in the tetrad stage, $37.1 \%$ in the uninucleate stage, at this dimension there are no microspores in the binucleated stage;

- in the case of V2 variant - the size of the buds $2.6-3.0 \mathrm{~mm}$, in the tetrad stage there are $27.8 \%$ of the microspores, uninucleated $60.2 \%$, and in the binucleated stage $1.9 \%$ (Figure 1);

- in the case of the V3 variant - the size of the buds 3.1-3.5 mm, the range of the microspores found in the tetrad stage decreases, reaching $1.1 \%$ of the total microspores, $90 \%$ of them are in the uninucleate stage, mainly in the stage traditionally uninucleated, while $2.8 \%$ are in the binucleated stage;

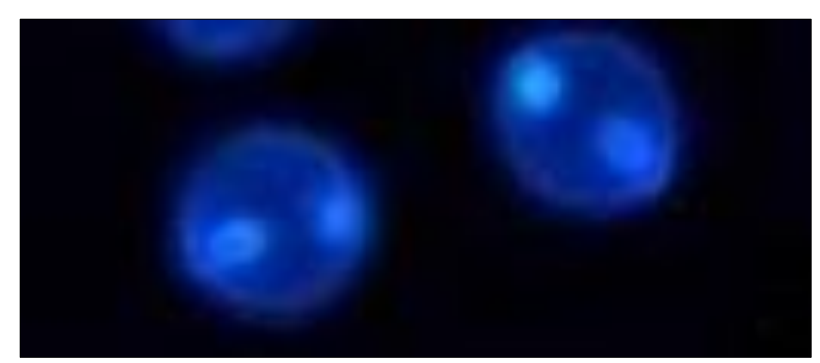

Figure 1. Microspores in binucleated stage

- In the case of the V4 variant - the size of the buds 3.6-4 mm, the tetrad stage disappears completely, the percentage of micropores in the uninucleated stage decreases to $51.8 \%$, concomitant with the increase of the percentage of micropores in the binucleated stage.

The first divisions of the microspores are visible from the first week after their incubation, gradually the cultures of microspores gaining a heterogeneous appearance, revealing both microspores with the gametophytic evolution (Figure 2) (transformation into pollen grains) as well as microspores with embryogenic competences ( 2 divided cells, surrounded by exine).

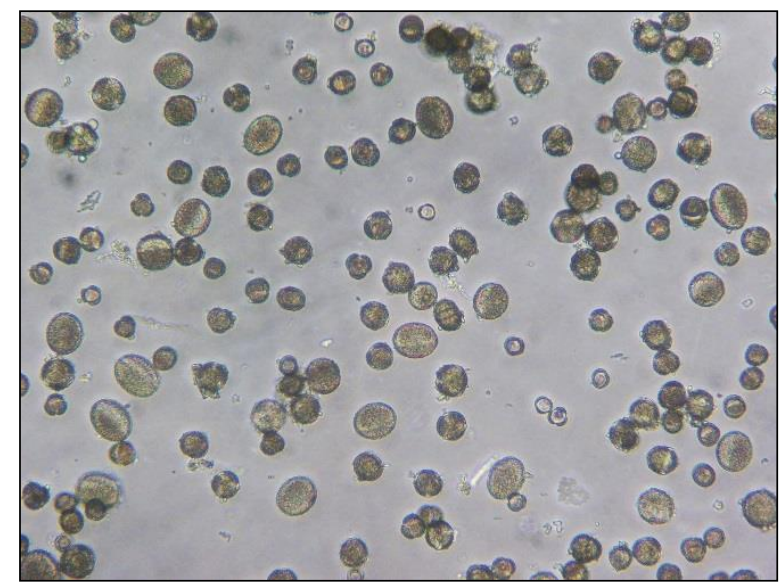

Figure 2. Heterogenous culture of microspores

Also, microspores that were injured during the isolation and washing operations were identified, as well as the microspores were completely devoid of morphogenetic reaction, which as time passed underwent transformations translated by lysis and cell death respectively (Table 3 ). 
Table 3. Type of morphogenetic reaction in isolated microspore culture at Brassica oleracea $\mathrm{l}$.

\begin{tabular}{|c|c|c|c|c|}
\hline Genotype & V1 & V2 & V3 & V4 \\
\hline BR121 & - & ++ & +++ & ++ \\
\hline
\end{tabular}

+++ more than $10 \%$ embryogenic microspores;

++ embryogenic cells and alive microspores;

+ embryogenic cells and dead microspores,

- no embryogenic cells, plasmolyzed microspores.

After the transfer to fresh culture media, the evolution of the embryogenic microspores continued, through the evolution stages similar to the somatic embryogenesis, finalized with the obtaining of the embryos. Initially after heat treatment, some of the microspores became embryogenic. The embryogenic microspores had several small vacuoles, and were usually bigger in size than the non-embryogenic microspores. The embryogenic microspores further divided and transformed in multinucleated microspores and multicellular embryos, still enclosed in the microspore wall can be observed. After 23 days, the exine wall is broken through and immature embryos appears.

Embryos in the cotyledons stage are taken from culture, under sterile conditions, at the laminar airflow niche and placed on solid culture media B5 [9]. To ensure the development of the embryos, the culture vials were incubated in climatic chambers, under photoperiod conditions $16 \mathrm{~h}$ light, at a temperature of $24^{\circ} \mathrm{C}$ and a luminous intensity of 5000 lux.

The results obtained are presented in Figure 3.

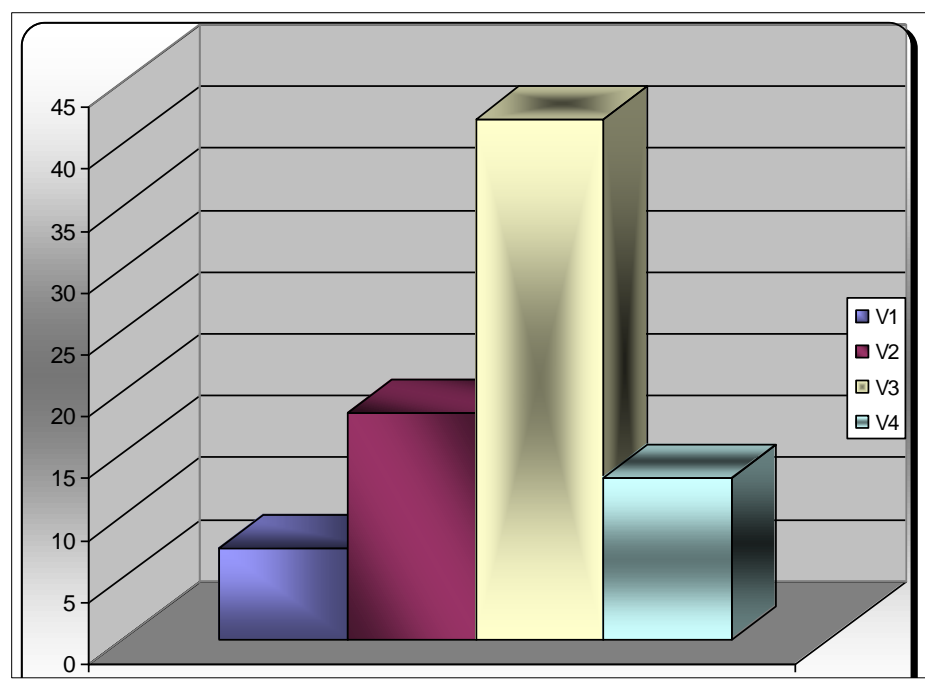

Figure 1. Graphical representation of average number of embryos obtained on the four experimental variants

After the statistical analysis of the results it can be emphasized that the best results were obtained in the case of using as a source of microspores the flower buds with the size between 3.1-3.5 mm. At this dimension, the share of microspores in the uninucleate stage, predominantly in the late uninucleate stage, is $90 \%$, thus ensuring a homogeneous population of microspores in the optimum stage of development. Their evolution is predominantly embryogenic, the percentage of microspores following the gametophytic pathway is reduced, by only $9.12 \%$.

\section{Conclusions}

For a successful protocol of microspore culture, the course of microspores evolution is switched, and forced to develop in a sporophytic way instead than the usual gametophytic pathway. Thus, identifying the size of flower bud that contains microspores in the proper developmental stage is crucial for successful microspore culture. Our results shows that the optimum dimension of floral buds is 3.1-3.5 $\mathrm{mm}$. At this size, the proportion of microspores in the uninucleate stage, predominantly in the late 
uninucleate stage, is $90 \%$, thus ensuring a homogeneous population of microspores in the optimum stage of development.

Acknowledgements. This work was cofinanced from the European Social Fund through the project: ADER 25.2.2: „Cercetare cu privire la proiectarea unui echipament inteligent horticol de analiză, predicție și acțiune biodinamică”, grant of the Romanian Ministry of Research and Innovation, CCCDI - UEFISCDI, project number PN-III-P1-1.2-PCCDI-2017-0850/ contract 14 PCCDI /2018, within PNCDI III, The BRESOV project GA no 7742 44, European Union's HORIZON 2020 research and innovation program.

\section{References}

1.CAO, M. Q.; LI, Y.; LIU, F.: JIANG, T., LIU, G. S. Application of another culture and isolated microspore culture to vegetable crop improvement. Acta Hort.,1995, 392, p. 27-38.

2.DUNWELL JM., Haploids in flowering plants: origins and exploitation. Plant Biotechnol J , 2010, 8, p. 377-424.

3.LICHTER R., Induction of haploids plants from isolated pollen of Brassica napus. Z. pflanzenphysiol., 1982, 105, p. 427-434 (1982).

4.PINK, D.: Application of Doubled Haploid Technology and DNA Markers in Breeding for Clubroot Resistance in Brassica oleracea. COST-824 Gametic Embryogenesis Workshop, Book of Abstracts, 57. Krakow, Poland. (1999).

5.WANG, M., FARNHAM, M.W., AND NANNES, J.S.P., Ploidy of broccoli regenerated from microspore culture versus anther culture. Plant Breeding, 1999, 118, p. 249-252.

6.BAILlIE, A. M. R.; EPP. D. J.: HUCHESON, D.: KELLER, W. A. In vitro culture of isolated microspores and regeneration of plants in Brassica campestris. Plant Cell Rep., 1992, 11, p. 234-237.

7.BARM. F.; MARTIN, A. Response of different genotypes of Brassica carinata to microspore culture. Plant Breed., 1999, 118, p.79-81.

8.WANG TT, LI HX, ZHANG J, OUYANG B, LU Y, YE Z., Initiation and development of microspore embryogenesis in recalcitrant purple flowering stalk (Brassica campestris ssp. chinensis var. purpurea Hort) genotypes. Sci Hort, 2009, 121, no. 4, p. p. 419-424.

9.GAMBORG, O. L., Plant tissue culture. Biotechnology. Milestones. In vitro Cellular and Developmental Biology - Plant, 1968, 38, p. 116-24.

10.HALE, A. L., M. W. FARNHAM, M. NDAMBE NZARAMBA, AND C. A. KIMBENG, Heterosis for horticultural traits in broccoli. Theor. Appl. Genet., 2007, 115, p. 351-360.

11.PECHAN, P. M., AND P. SMYKAL, Androgenesis: affecting the fate of the male gametophyte. Plant Physiol, 2001, 111, p. 1-8.

Manuscript received: 17.12 .2019 